\title{
Influence of the impact energy on the pattern of blood drip stains
}

\author{
F. R. Smith, ${ }^{1, *}$ C. Nicloux,${ }^{2}$ and D. Brutin ${ }^{1,3, \dagger}$ \\ ${ }^{1}$ IUSTI, UMR No. 7343, Aix-Marseille University, 13013 Marseille, France \\ ${ }^{2}$ Institut de Recherche Criminelle de la Gendarmerie Nationale, 95300 Pontoise, France \\ ${ }^{3}$ Institut Universitaire de France, 75231 Paris, France
}

(Received 20 September 2017; published 11 January 2018)

\begin{abstract}
The maximum spreading diameter of complex fluid droplets has been extensively studied and explained by numerous physical models. This research focuses therefore on a different aspect, the bulging outer rim observed after evaporation on the final dried pattern of blood droplets. A correlation is found between the inner diameter, the maximum outer diameter, and the impact speed. This shows how the drying mechanism of a blood drip stain is influenced by the impact energy, which induces a larger spreading diameter and thus a different redistribution of red blood cells inside the droplet. An empirical relation is established between the final dried pattern of a passive bloodstain and its impact speed, yielding a possible forensic application. Indeed, being able to relate accurately the energy of the drop with its final pattern would give a clue to investigators, as currently no such simple and accurate tool exists.
\end{abstract}

DOI: 10.1103/PhysRevFluids.3.013601

\section{INTRODUCTION}

A lack of adequate scientific validity in some forensic methods has been pointed out [1]. In order to prevent wrong convictions due to doubtful interpretation of forensic evidence, some governments are currently strengthening and promoting a more rigorous approach to forensic science [2]. In this respect, the study of bloodstains is divided into three categories, passive, altered, or projected blood spatters. We focus here only on passive traces by examining links between the final pattern and the nature of an impact of a drop dripping naturally. Indeed, the effect of gravity on a blood mass induces the mass to release droplets which will next travel through the air while retaining a spherical shape due to surface tension. The drop then impacts a surface at an angle of $90^{\circ}$ and a circular stain is induced. The present work is devoted to cases of blood droplets dripping only due to gravity, without any initial velocity. The initial kinetic energy would modify the final impact energy and it would then not be possible to recalculate the initial height of the fall.

A previous study by Brutin et al. [3] demonstrated the similarities between a deposited sessile whole blood droplet and a colloidal suspension droplet since blood is a colloidal fluid. The red blood cells (RBCs) constitute the main colloidal component which forms cracks during the drying process. The drip stain dries following two different regimes and five different stages. The first regime is driven by convection, diffusion while coagulation takes place, and then gelation (first three stages). At the time of drop deposition, RBCs are evenly distributed inside the drip stain and then the evaporation flux at the interface induces an internal flow that transports the particles inside the drop. When the particle concentration reaches a critical point a gel forms and a biological deposit is observed on the periphery of the drip stain. Once the entire drop gels, which corresponds to the transition phase,

\footnotetext{
*fiona.smith@univ-amu.fr
}

†david.brutin@univ-amu.fr 


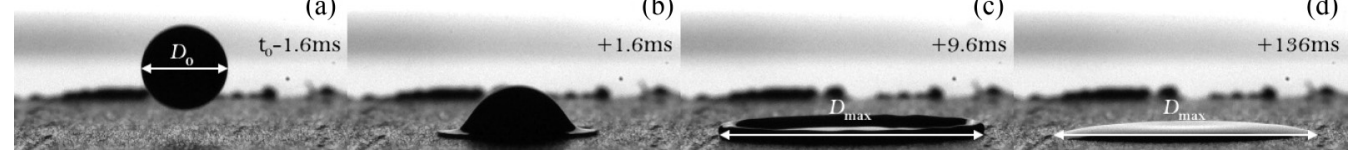

FIG. 1. Blood droplet impact recorded with a high-speed camera $\left(U=1.85 \mathrm{~m} \mathrm{~s}^{-1}\right.$ and $\left.D_{0}=3.34 \mathrm{~mm}\right)$. (a) Blood droplet before its impact showing its initial diameter $D_{0}$. (b) Impact and spreading of the droplet. (c) Spreading reaches its maximum spreading diameter $D_{\max }$. (d) The capillary and viscous forces act on the droplet, but the maximum diameter is unchanged due to self-pinning, and now evaporation starts.

a sharp decrease of the drying rate is observed. This slower second regime is diffusive and finally, the last two stages involve the formation of cracks through nucleation and propagation. The drying stages of deposited blood droplets were thus extensively explained by this work, and some interesting features were already highlighted, mainly the formation of a thicker rim observed throughout drying. A later work by Laan et al. [4] described the spreading of blood droplets by presenting a model that explains the impact behavior of fluids on solid surfaces. The spreading is driven by inertia but is countered by capillary and viscous forces $[5,6]$ as shown in Fig. 1. Commonly, these forces are expressed in the form of ratios: the Weber number ( $\left.\mathrm{We}=\rho D_{0} U^{2} / \sigma\right)$ between capillary and inertial forces and the Reynolds number $\left(\operatorname{Re}=\rho D_{0} U / \eta\right)$ between the viscous and the inertial forces. Here $\rho$ and $\eta$ are the blood density and viscosity, respectively, $\sigma$ is the blood-air surface tension, and $U$ stands for the impact velocity of the droplet. The Laan et al. model was later corrected by the model of Lee et al. [7] that describes the spreading of all liquid on rough and smooth surfaces. It is governed by energy conservation and this model introduces the rescaled spreading ratio as a function of the Weber number. Hence, from this solution it is possible to find the impact velocity $U$ as it will alter the maximum spreading ratio, but an initial piece of information, namely, the initial diameter of the droplet or its volume, is required. In order to complete this approach, as well as other approaches that already exist [8-10], a different parameter is thus necessary to determine the impact velocity from the final bloodstain. However, it could be assumed that the impact velocity, because of altering the maximum spreading diameter, will have an influence on the drying stage, mainly on the internal flow observed during the first regime and will affect the observed pattern of the drip stain. Accordingly, the RBCs distribution would be altered as well as the biological deposit leading to a noteworthy change in the width of the bulging outer rim and a small change between the maximum spreading diameter of the droplet at impact and the outer diameter of the same drip stain after drying. Thus, by correlating the impact velocity and the outer diameter of the blood droplet with this parameter, the bulging outer rim width giving this characteristic doughnut shape, we aim to provide a solution that would allow us to estimate the impact velocity of a dried blood drip stain.

\section{METHOD AND MATERIALS}

\section{A. Experimental setup and uncertainties}

To test different impact energies, we used 16 different heights of fall ranging from 0.6 to $152 \mathrm{~cm}$ and two needle diameters, one of $25 \mathrm{G}(0.5080 \mathrm{~mm}$ in diameter $)$ and a second of $18 \mathrm{G}(1.219 \mathrm{~mm}$ in diameter). For each case we repeated the experiment twice. Hence, the impact velocities ranged between 0.19 and $4.83 \mathrm{~m} \mathrm{~s}^{-1}$ and the initial diameters of the droplets were from $2.33 \times 10^{-3}$ to $3.46 \times 10^{-3} \mathrm{~m}$. The substrate used was wood flooring since it is a substrate encountered very often on crime scenes. Blood wets this surface with a contact angle of $80.0^{\circ} \pm 7.29^{\circ}$, which suggests that we place ourselves in the case of a partially wetting substrate. The droplets were released into a humidity of $(30 \pm 5) \%$ on average and were then left to dry in a glove box (Jacomex T-Box, $V=350 \mathrm{~L}$ ) at a constant humidity of $(30 \pm 2) \%$. To record the impacts of droplets, we used a high-speed camera (Fastcam-ultima 1024). To measure the volume of the droplet, the initial diameter of the droplet, its speed at the time of the impact, and its maximum spreading diameter we used the software IMAGEJ to 
analyze the pictures recorded with the high-speed camera. After drying, one droplet for each different case was then scanned with a confocal microscope (STIL Micromesure 2, with two different optical sensors: 4000 m CCS Prima n2 and $150 \mathrm{~m}$ CCS Prima) in order to obtain the three-dimensional (3D) profile of the dried droplets. Scans lasted on average $5 \mathrm{~h}$. A final picture of the dried droplet was then taken with a camera (Canon EOS 7D digital camera, resolution $5184 \times 3456$ pixels). To quantitatively measure the bulging outer rim dimension width, we measured both the inside diameter $D_{2}$ and the maximum outer diameter $D_{1}$ with scanning probe image processor software. With pictures taken and the radial cross sections from the 3D profile visualization of the droplet, it was possible to identify the best feature that would allow us to evaluate the internal diameter, the parameter on which our study focuses. The picture provides an optical tool for easy monitoring at a crime scene, but it was important to associate both approaches in order to set up a protocol that can be easily reproduced later on. On the 3D profile, we observed a decrease of the profile heights and noticeable features which when compared to the pictures corresponded to what we would visually identify as the bulging outer rim.

\section{B. Blood properties}

To create several drops of blood with different impact energies, we sampled blood from healthy volunteers, of a sample group of adult women and men of different ages, stored it in sterile tubes coated with ethylenediamine tetra-acetic acid (EDTA) anticoagulant (BD Vacutainer 4-mL tubes spray coated with $K_{2}$ EDTA), and kept them in a refrigerator at $5{ }^{\circ} \mathrm{C}$. The blood was then used within two days to avoid blood aging and an important decrease of platelets that starts being noticeable after two days [11]. Indeed, RBCs and platelets cannot be conserved at the same temperature: RBCs are conserved at a temperature of $5^{\circ} \mathrm{C}$ and platelets between $20^{\circ} \mathrm{C}$ and $24^{\circ} \mathrm{C}$. For the same person, results were consistent, emphasizing that storage conditions were good and that blood aging was not affecting the results. Before being used, the blood was reheated at room temperature and swirled for $10-15 \mathrm{~min}$. The blood was then collected with a $200-\mu \mathrm{L}$ Eppendorf pipette. A new needle and a new plastic tip were used for every new blood collection in order to avoid blood aggregation. Hematologic analysis (Mindray BC 3600) was performed, giving precise information concerning blood compositions, namely, their hematocrit concentrations that were comprised in the range from $(34.2 \pm 0.1) \%$ to $(37.6 \pm 0.1) \%$ for women and from $(42.1 \pm 0.1) \%$ to $(47.1 \pm 0.1) \%$ for men. This contributes to the known small blood density variation between 1020 and $1060 \mathrm{~kg} \mathrm{~m}^{-3}$. Taking into account that this density variation is less than $4 \%$, a constant density of $\rho=(1040 \pm 20) \mathrm{kg} \mathrm{m}^{-3}$ was assumed for the rest of the study. Similarly, the viscosity and the surface tension of blood are required to consider the physical behavior of blood droplets. Studies agree with the statement that blood has viscoelastic and shear thinning properties of non-Newtonian fluids, although it spreads like Newtonian fluids. As Kolbasov et al. mentioned in their study [12], the case of blood dripping from a weapon involves the elongated shear component flow. Thus the high-shear viscosity $\eta=4.8 \mathrm{mPa} \mathrm{s}$ and the blood surface tension average value $\sigma=69.8 \mathrm{mN} \mathrm{m}^{-1}$ are assumed as well for the rest of the study.

\section{RESULTS AND DISCUSSION}

The aim of the research is to find the relationship between the final observed pattern and its velocity $U$ at the time of the impact. To do so, we choose to first validate our data with already existing spreading models for droplets. The first model presented by Clanet et al. [13] depends on mass and momentum conservation and predicts that the maximum spreading $D_{\max }$ scales as $D_{0} \mathrm{We}^{1 / 4}$ using an impact parameter $P=\mathrm{We} \mathrm{Re}^{-4 / 5}$ as shown in Fig. 2. This states that the spreading of droplets is limited either by capillarity or by viscosity and that two distinct regimes exist, a capillary regime for $P<1$ and a viscous regime for $P>1$.

The second approach of Lee et al. [7] presented in Fig. 3 relates capillary, viscosity, and inertia forces according to the Eggers et al. [14] approach. In order to do so we quantitatively relate 


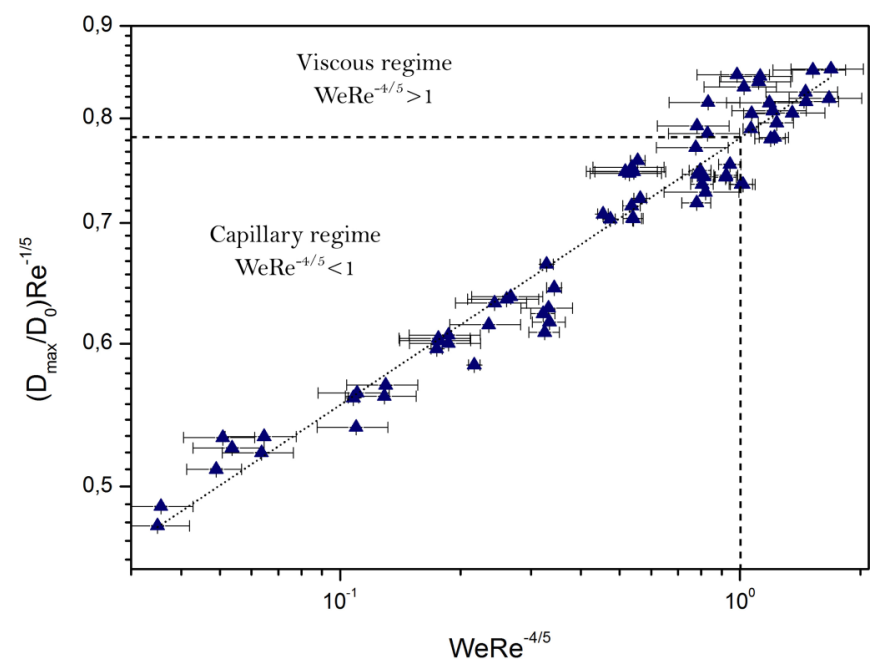

FIG. 2. Rescaled maximum spreading ratio as a function of $\mathrm{We} \mathrm{Re}^{-4 / 5}$ according to the solution proposed by Clanet et al. [13] for maximum spreading diameters of droplets on solid substrates.

the maximum spreading diameter to the impact velocity, which takes into account that energy conservation is the only physical principle required to explain droplet spreading behavior. This model uses the Padé approximation

$$
\left(\beta_{\max }^{2}-\beta_{0}^{2}\right)^{1 / 2} \mathrm{Re}^{-1 / 5}=\frac{\mathrm{We}^{1 / 2}}{A+\mathrm{We}^{1 / 2}},
$$

with the fitting constant $A=7.6$. The approach describes the correlation that exists between the maximum diameter of the spreading droplets and the velocity for a nonporous substrate. This model provides satisfactory universal scaling, as can be seen in Fig. 3, and a clear explanation of the phenomenon that takes place. A more recent study accounts for the energy involved and the dissipation

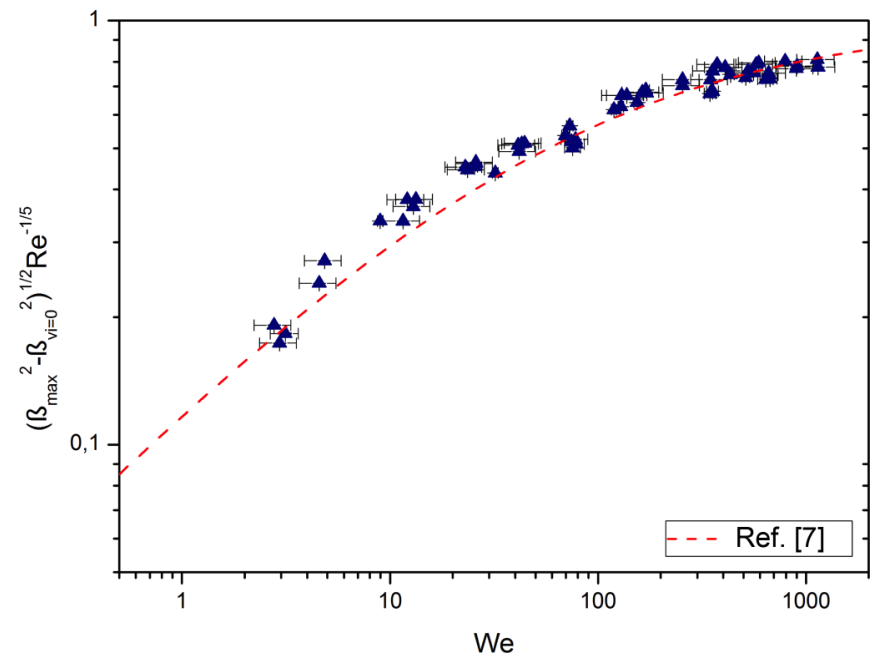

FIG. 3. Rescaled maximum spreading ratio as a function of Weber number according to universal solution proposed by Lee et al. [7] for maximum spreading diameters of droplets on solid substrates. 


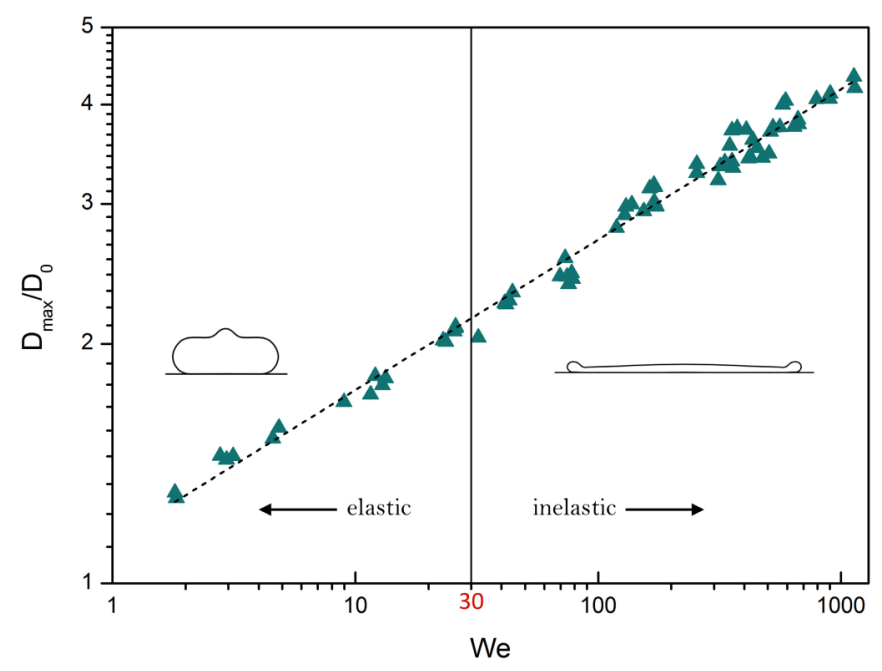

FIG. 4. Rescaled maximum spreading ratio as a function of Weber number according to the solution presented by Wildeman et al. [15]. The dashed line shows the tendency that our experimental data set follows, which is similar to the no-slip model in the inelastic regime.

mechanisms occurring during droplet impact on solid surfaces [15]. This simpler model offers to distinguish two behaviors, an elastic one for small Weber numbers, less than 30, and an inelastic one for Weber numbers greater than 30, as shown in Fig. 4. As expected, our set of experimental data follows the no-slip model. The Ohnesorge number $(\mathrm{Oh}=\sqrt{\mathrm{We}} / \mathrm{Re})$ and the $K\left(K=\mathrm{We}^{1 / 2} \mathrm{Re}^{1 / 4}\right)$ parameter are other dimensionless numbers important in the description of the drop impact pattern. Nonetheless, as some recent works on liquids [16] and more specifically on blood [17] explained, these parameters are essentially used to describe delineation and splashing regimes. In this study, the aim is to relate the impact velocity with the impact pattern, without knowing the initial volume of the droplet. We thus focus on providing a complementary parameter, different from the maximum spreading ratio, by introducing a feature that can be observed on dried drip stains. As described by Ramsthaler et al. [18], the shape of a droplet is defined due to the evaporation from the contact line pinning effect arising from the liquid-air and substrate-liquid interfacial tensions. A blood droplet follows the constant base model, which assumes that the outer diameter of the droplet remains constant while the contact angle decreases. The behavior induces a decrease in the profile height as well, but this is limited due to the presence of particles, in our case RBCs. It was noticed that in the case of suspensions, the height stops decreasing when the suspension gels. As explained previously, the different drying regimes of a blood droplet and internal flow induce the RBCs to be driven to the periphery of the droplet until gelation of the droplet occurs. After drying we observed that on the final pattern of dried droplets, a circular bulge was visible around its rim, as shown in Fig. 5. Additionally, these visual observations reveal that the width of the bulging outer rim, corresponding to the distance between the inside diameter and the maximum spreading diameter of the droplet, varies depending on the impact energy. This bulging outer rim seen on the final pattern is the biological deposit that forms during drying due to the internal flow driving the RBCs to the periphery of the droplet, which corroborates our previous assumption that the internal flow is modified by the impact velocity. Dried blood drip stains exhibit the well-known coffee-ring stain shape. This singular shape is the result of the time-scale competition between the thermocapillary convection and the movement of the particles. As Deegan et al. described [19], a strong capillary flow and a weak Marangoni flow are responsible for a successful coffee-ring shape, since, to observe this shape, the evaporation cannot be faster than the movement of the particles towards the three-phase contact line. Figure 6(a) shows how the internal flow tends to replenish water evaporating from the edges, inducing the RBCs to be displaced 
(a)
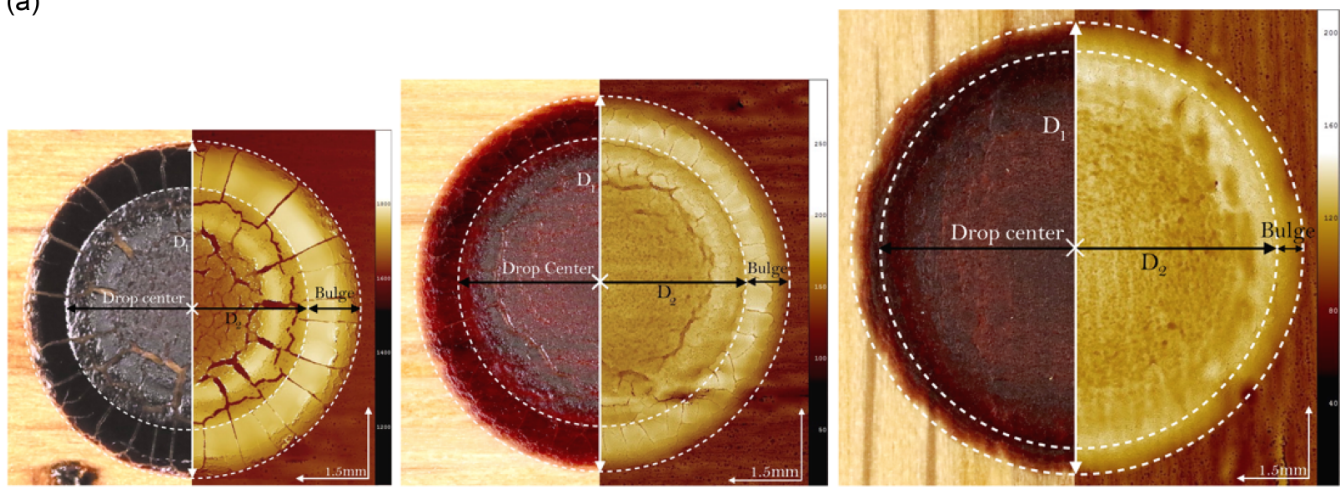

(b)

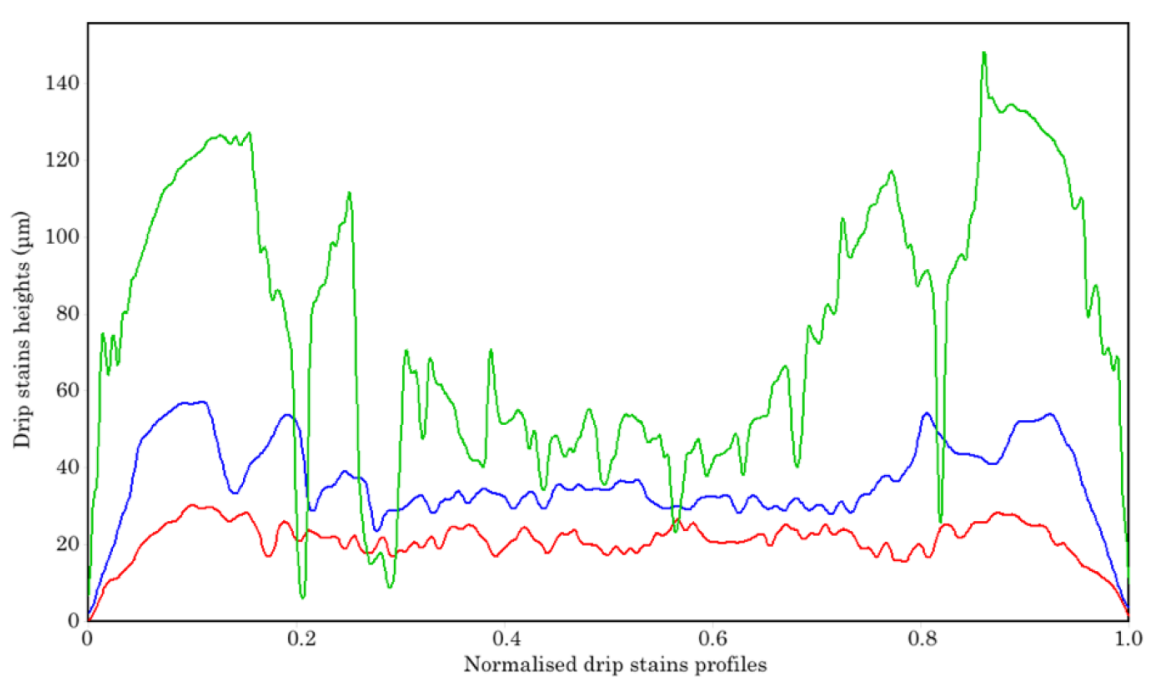

FIG. 5. (a) View of dried blood drip stains with bulging outer rim, from a healthy volunteer. Droplets are of initial diameters $D_{0}=2.4 \mathrm{~mm}$ and impact velocities, from left to right, of $0.74,1.87$, and $4.75 \mathrm{~m} \mathrm{~s}^{-1}$. On the left side with photography and on the right side with confocal microscopy that gives the $3 \mathrm{D}$ features by means of radial cross sections. (b) Cross-section profiles of three droplets of the same volume with impact velocities of 0.74 (green), 1.87 (blue), and $4.75 \mathrm{~m} \mathrm{~s}^{-1}$ (red).

towards the rim of the droplet forming after evaporation, leading to the observed bulge. Figure 6(b) shows the changes observed for impacting blood droplets with higher impact velocity. This added kinetic energy for the same volume leads to a larger spreading of the droplet and a decrease in its thickness. The evaporation is thus faster and preferred at the contact line, which generates a surface tension gradient that drives a stronger recirculation, namely, Marangoni flow, and for the RBCs to homogenize. In that case, a clear coffee-ring effect cannot be successfully observed since the water evaporates faster and the RBCs movement towards the periphery of the droplet is weaker. Hence, in the case of a droplet impacting at higher velocity for the same volume, the thinning of the drip stain thickness induces a faster evaporation and a change in the internal flows. The $3 \mathrm{D}$ profile visualization of the dried bloodstain was used to characterize the features marking the rim dimensions in a radial cross section. Thus we were able to record the internal diameter of each droplet as shown in Fig. 5, by averaging its measurement in different radial cross sections and comparing it to photographic analysis. 
(a) Low impact velocity

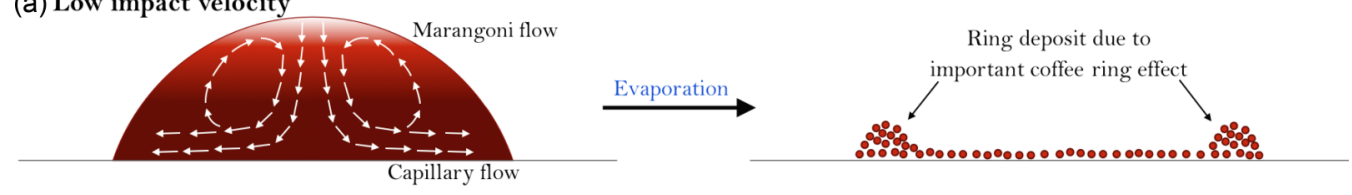

(b) High impact velocity

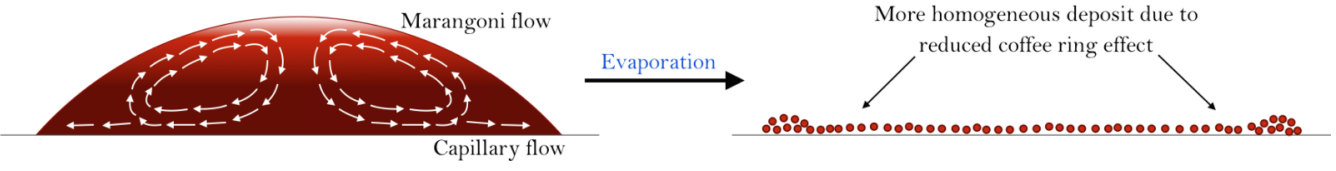

FIG. 6. (a) Motion of RBCs under standard ambient evaporation conditions inside a drip stain that impacted at low velocity. Differential evaporation rates at the edge and in the middle drive the particles to the edges, leading to coffee-stain formation. (b) Motion of RBCs undergoing the influence of Marangoni flows for drip stains that impacted at higher velocities. As previously, RBCs tend to move toward the edges, but a higher surface tension on top engenders a Marangoni flow from the contact line toward the top of the droplet. This induces a recirculating flow pattern as represented above. The recirculating flow homogenizes the particle concentration, overcoming coffee-stain formation and leading to a more uniform deposition.

From these measurements, we can introduce a parameter $D_{2}$, which corresponds to the observed internal diameter: the diameter of the droplet excluding the bulging outer rim. A slight shrinkage or postimpact spreading were observed during the drying processes of the droplets. Minor shrinkage was observed for droplets falling with higher impact energy, due to the Marangoni flow, whereas spreading was observed with droplets of low impact energy, this time due to the capillary flow. We compared the maximum outer diameter after drying to the one obtained with the fast camera and observed variations ranging from $88 \%$ to $127 \%$, in a progressive manner with slower impact speeds. We thus refer to $D_{1}$ as the maximum outer diameter of the drip stain after drying. We then used a different approach in order to observe the influence of the impact on the spreading of both diameters. To do so, we chose to observe a commonly used parameter, the spreading ratio [20].

We expressed both diameters as spreading ratios $\beta_{1}=D_{1} / D_{0}$ and $\beta_{2}=D_{2} / D_{0}$, with $D_{0}$ being the initial diameter of the droplet. To quantitatively find a relationship that could in turn work to back calculate the initial impact velocity of droplets knowing only $D_{1}$ and $D_{2}$, we used two different approaches. The first method is a direct approach relating the spreading ratio as a function of the impact velocity $U$ as shown in Fig. 7(a).

The first approximation function in Fig. 7 which relates $\beta_{1}$ to $U$ is given by

$$
\beta_{1}=a U^{b}
$$

with $a=2.34$ and $b=0.374$. This is obtained by means of a least-squares fit $\left(R^{2}=0.960\right)$. The second approximation function relating $\beta_{2}$ to $U$ is

$$
\beta_{2}=c U^{d},
$$

with $c=1.66 \mathrm{~m}$ and $d=0.487$, also obtained by means of a least-squares fit $\left(R^{2}=0.966\right)$. We assume that these evaluated constants are substrate dependent and correspond to the wooden flooring used during our experiment. The internal natural convection of the drop is thus widely influenced by the spreading at the time of the impact, which changes its internal mechanical properties. Thus this accumulation of biological elements at the edge of the droplet leading to the bulge formation gives us a feature that can be exploited to find information on the initial state of the falling droplets. Moreover, this feature is found on dried patterns, which is relevant from a forensic point of view, as drip stains would be dried at the time of the investigation. With this piece of information we can calculate the initial velocity $U$ from both fitted functions (2) and (3) by correlating them together; 

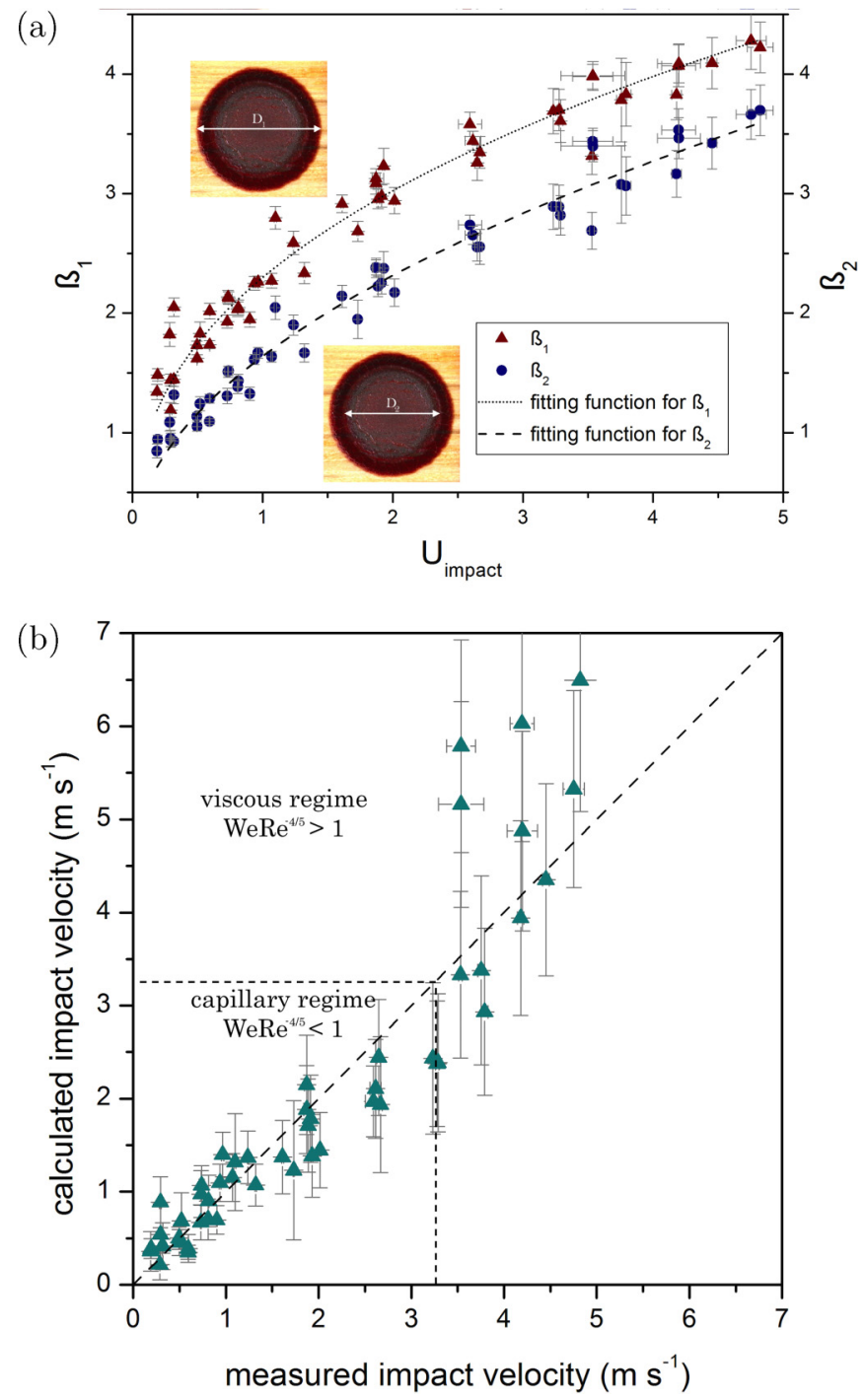

FIG. 7. (a) Maximum spreading ratio as a function of $U$ (in red) and its fitted function, and internal spreading diameter as a function of $U$ (in blue) and its fitted function. (b) Calculated impact velocity using Eq. (4) versus measured impact velocity.

for example, the expression obtained for the velocity is

$$
U=\left(\frac{D_{1}}{D_{2}} \frac{c}{a}\right)^{1 /(b-d)} .
$$

We then compared the calculated impact velocity with the measured one as shown in Fig. 7(b). We observed that this proposed model is accurate for droplets impacting the surface at low velocities, below $3 \mathrm{~m} \mathrm{~s}^{-1}$, but overestimates the calculated velocity for droplets impacting at high velocities. This observation on blood droplets supports the study of Clanet et al., who showed that the maximum spreading of low-viscosity droplets may be limited either by capillarity or by viscosity, presenting thus two distinct regimes: one at low impact velocity, which corresponds to the capillary regime, and one at high impact velocity, corresponding to the viscous regime [13]. In our case we observe 

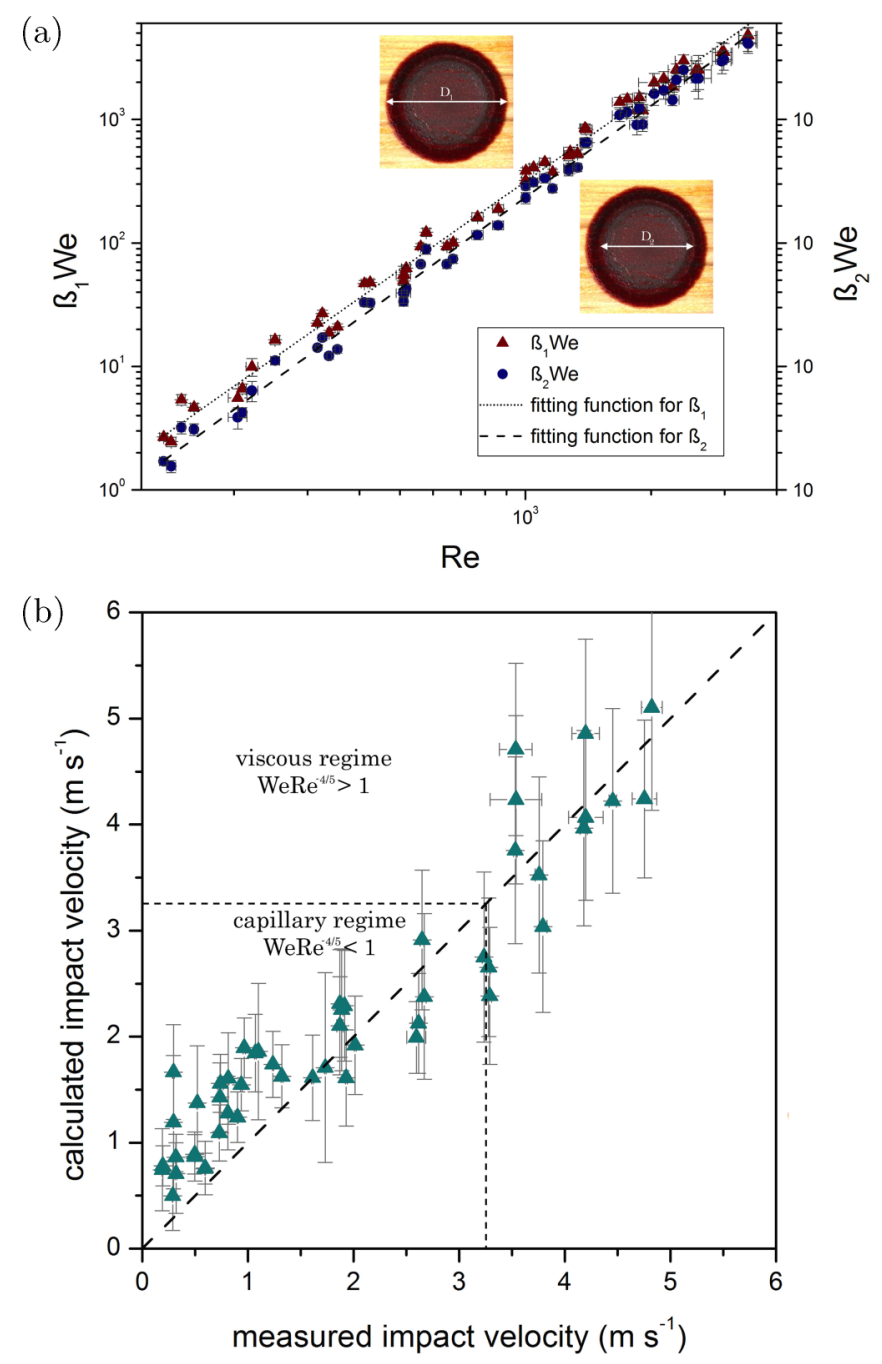

FIG. 8. (a) Rescaled maximum spreading ratio as a function of Re (in red) and its fitted function, and rescaled internal spreading diameter as a function of Re (in blue) and its fitted function. (b) Calculated impact velocity using Eq. (9) versus measured impact velocity.

as well that different regimes affect the internal flows and that the first solution correlating directly the impact velocity with the spreading ratios works well in the capillary regime. Hence, in order to find a more adequate solution for droplets in the viscous regime, more parameters, such as viscosity, need to be taken into account; we thus correlated both diameters $D_{1}$ and $D_{2}$ with the Weber and Reynolds numbers. We note that these two diameters, when multiplied with their corresponding Weber numbers that account for the inertia forces in the spreading of the droplet and the superficial tension with the substrate, are functions of their Reynolds numbers. Figure 8 (a) shows $\beta_{1}$ We as a function of Re and $\beta_{2}$ We as a function of Re.

The first approximation function in Fig. 8 which relates $\beta_{1}$ We to Re is given by

$$
\beta_{1} \mathrm{We}=a^{\prime} \operatorname{Re}^{b^{\prime}}
$$


with $a^{\prime}=3.90 \times 10^{-4}$ and $b^{\prime}=2.00$. This is obtained by means of a least-squares fit $\left(R^{2}=0.983\right)$. The second approximation function relating $\beta_{2}$ We to Re is

$$
\beta_{2} \mathrm{We}=c^{\prime} \operatorname{Re}^{d^{\prime}},
$$

with $c^{\prime}=1.20 \times 10^{-4}$ and $d^{\prime}=2.13$, also obtained by means of a least-squares fit $\left(R^{2}=0.983\right)$.

Again, we assume that these evaluated constants are substrate dependent and correspond to the wooden flooring used during our experiment. Once more we want to calculate the impact velocities of the droplets by correlating the fitted functions (5) and (6). To do so we can express first the Reynolds number with our functions as

$$
\operatorname{Re}=\left(\frac{D_{1}}{D_{2}} \frac{c^{\prime}}{a^{\prime}}\right)^{1 /\left(b^{\prime}-d^{\prime}\right)} .
$$

Then we express the Weber number over $D_{0}$ with the calculated Reynolds number

$$
\frac{\mathrm{We}}{D_{0}}=a^{\prime} \frac{\operatorname{Re}^{b^{\prime}}}{D_{1}} .
$$

Finally, we can recalculate the impact velocities and compare them to the measured ones, as shown in Fig. 8(b), due to the expression of the Weber number

$$
U=\sqrt{\frac{\mathrm{We}}{D_{0}} \frac{\sigma}{\rho}}=\sqrt{a^{\prime} \frac{\operatorname{Re}^{b^{\prime}}}{D_{1}} \frac{\sigma}{\rho}}=\sqrt{\left(\frac{c^{\prime}}{D_{2}}\right)^{b^{\prime} /\left(b^{\prime}-d^{\prime}\right)}\left(\frac{D_{1}}{a^{\prime}}\right)^{d^{\prime} /\left(b^{\prime}-d^{\prime}\right)} \frac{\sigma}{\rho}} .
$$

We observe that this model offers an overall better solution, especially concerning droplets in the viscous regime, but tends to overestimate the calculated velocities of droplets that are in the capillary regime. Although this model takes into account viscosity, density, and surface tension, blood is a non-Newtonian fluid and the changes in viscosity are not taken into account. The shear stressed induced on blood droplets cannot be measured; a constant high shear rate blood viscosity is used in Fig. 8. Accordingly, the viscosity of blood droplets impacting at low velocity is overestimated, which explains the corresponding overestimation of velocity back calculations. This implies that this model would offer a more accurate solution than the first model for Newtonian fluid since the viscosity remains constant, but for blood both models are necessary. By introducing the internal diameter as an alternative measurable feature of a spreading blood droplet, it then becomes possible to relate the dried pattern of a drip stain with its initial velocity. From these observations, it can be established that the impact energy arising from the fall of the droplet induces a larger spreading that affects the internal flow and RBCs are proportionally less spread from the center of the drop towards the periphery but are more evenly distributed, as the ratio $D_{2} / D_{1}$ decreases with impact velocity. The biological deposit becomes less noticeable as the impact energy increases and we can assume that the center of the droplet is richer in RBCs; further, since throughout the droplet the volume distribution tends to be more even, it suggests that drip stains during desiccation are less subjected to stress. This stress normally leads to the cracking phenomenon observed during the drying of sessile droplets. However, we observed that for droplets with higher impact velocity, cracks were less noticeable, as shown in Fig. 5, and moreover the drip stains were not peeling off the substrate, unlike drip stains with low velocities. From our experimental recordings, we can consequently correlate the impact velocity with the final dried pattern of the bloodstain. Due to this calculated impact velocity, it is possible to give an estimated range height from which the drop would be coming from. This model thus complements previous known models by showing features that provide the missing information that was needed to give and estimate the velocity of impact of dried drip stains such as the ones encountered at crime scenes, though, as explained previously, this would be applicable only to drip stains arising from natural dripping. In the case of passive dripping from weapons, the initial velocity would be modified [21], thus the only possible estimation becomes then the maximum height of the fall. Since no distinction can be done between drops falling with or without any initial velocity if they 
hit the surface at a $90^{\circ}$ angle, the maximum height of the fall is the only evidence that can be deduced. Nonetheless, this already gives a significant piece of information in crime scene reconstruction by excluding some reconstitution patterns that would not fit with this evidence. For example, it could answer questions such as whether the person was standing up or kneeling. It is important to point out that this study was realized for one substrate, thus the constants $a, b, c, d$ and $a^{\prime}, b^{\prime}, c^{\prime}, d^{\prime}$ of the proposed models would be modified with another substrate and would depend not only on roughness, but on wettability as suggested by previous studies, such as the one of Sahu et al. that showed the influence of surface wettability on drop impact patterns in gravity dripping of aqueous suspensions [22]. In addition, we also excluded droplets with significant splashing, as it would change the volume of the droplet. However, we measured the maximum height at which splashing was observed with our substrate, testing different release heights at a humidity of $(30 \pm 5) \%$, which is susceptible to change for a different humidity [23]. We measured it to be $(2.1 \pm 0.1) \mathrm{m}$. A height of $2.1 \mathrm{~m}$ is greater than would be encountered in most forensic situations where this analysis has potential application. Finally, we used blood from healthy volunteers, and some studies [24] suggest that pathologies have an influence on the blood dried pattern.

\section{CONCLUSION}

As demonstrated, there is a correlation between the width of the bulging outer rim and the impact velocity, which is observed when measuring $D_{2}$ and $D_{1}$. The drying pattern of a deposited blood drip stain is already well explained by the literature, as is the maximum spreading ratio of dripping blood drops. However, this parameter enables us to understand how the observed final pattern is influenced by the kinetic energy of a blood droplet at the time of the impact and how can it be exploited in forensic applications. Indeed, by measuring the outer and internal diameters of a dried droplet after impact, we have quantified the influence of the impact velocity on the internal flow of a drying blood droplet. This kinetic energy induces a larger spreading and a thinning of the drip stain, which in turn provokes the RBCs distribution to be more evenly spread inside the droplet by affecting the drying flows. An increase of the internal diameter ratio is measured with a corresponding increase in velocity. At higher impact velocities the cracks are less evident as well due to a better redistribution of the RBCs and hence a reduced stress. This paper complements previous work on the spreading and drying of blood droplets, by giving a more complete view of the observed physical behavior. From a forensic point of view, this method presents a feature that could possibly be exported outside the laboratory context and tested on real crime scenes, as only a picture of a passive bloodstain would be needed. Then, depending on the regime of the blood droplet, one model should be preferred over another. The research should now be extended to other substrates, in order to subsequently present sets of coefficient values that could be used in forensic crime scene reconstitution.

\section{ACKNOWLEDGMENTS}

We would like to thank the volunteers who gave their blood and the nurse who sampled this blood. This work received a financial grant from the French Agence Nationale de la Recherche in the frame of Project No. ANR-13-BS09-0026. Also, this work was carried out in the framework of the Labex MEC Project No. ANR-10-LABX-0092 and of the A*MIDEX Project No. ANR11-IDEX-0001-02, funded by the Investissements d'Avenir French Government program managed by the French National Research Agency.

[1] K. G. de Bruin, R. D. Stoel, and J. C. M. Limborgh, Improving the point of origin determination in bloodstain pattern analysis, J. Forensic Sci. 56, 1476 (2011).

[2] B. Colglazier, Encourage governments to heed scientific advice, Nature (London) 537, 590 (2016). 
[3] D. Brutin, B. Sobac, B. Loquet, and J. Sampol, Pattern formation in drying drops of blood, J. Fluid Mech. 667, 85 (2011).

[4] N. Laan, K. G. de Bruin, D. Bartolo, C. Josserand, and D. Bonn, Maximum Diameter of Impacting Liquid Droplets, Phys. Rev. Appl. 2, 044018 (2014).

[5] I. V. Roisman, R. Rioboo, and C. Tropea, Normal impact of a liquid drop on a dry surface: Model for spreading and receding, Proc. R. Soc. A 458, 1411 (2002).

[6] H. Marmanis and S. T. Thoroddsen, Scaling of the fingering pattern of an impacting drop, Phys. Fluids 8, 1344 (1996).

[7] J. B. Lee, N. Laan, K. G. de Bruin, G. Skantzaris, N. Shahidzadeh, D. Derome, J. Carmeliet, and D. Bonn, Universal rescaling of drop impact on smooth and rough surfaces, J. Fluid Mech. 786, R4 (2016).

[8] C. D. Adam, Fundamental studies of bloodstain formation and characteristics, Forensic Sci. Int. 219, 76 (2012).

[9] D. Attinger, C. Moore, A. Donaldson, A. Jafari, and H. A. Stone, Fluid dynamics topics in bloodstain pattern analysis: Comparative review and research opportunities, Forensic Sci. Int. 231, 375 (2013).

[10] M. A. Raymond, E. R. Smith, and J. Liesegang, The physical properties of blood: Forensic considerations, Sci. Justice 36, 153 (2013).

[11] J. Riddel, B. Aouizerat, C. Miaskowski, and D. Lillicrap, Theories of blood coagulation, J. Pediatric Oncol. Nurs. 24, 123 (2007).

[12] A. Kolbasov, P. M. Comiskey, R. P. Sahu, S. Sinha-Ray, A. L. Yarin, B. S. Sikarwar, S. Kim, T. Z. Jubery, and D. Attinger, Blood rheology in shear and uniaxial elongation, Rheol. Acta 55, 901 (2016).

[13] C. Clanet, C. Béguin, D. Richard, and D. Quéré, Maximal deformation of an impacting drop, J. Fluid Mech. 517, 199 (2004).

[14] J. Eggers, M. A. Fontelos, C. Josserand, and S. Zaleski, Drop dynamics after impact on a solid wall: Theory and simulations, Phys. Fluids 22, 062101 (2010).

[15] S. Wildeman, C. W. Visser, C. Sun, and D. Lohse, On the spreading of impacting drops, J. Fluid Mech. 805, 636 (2016).

[16] A. L. Yarin, I. V. Roisman, and C. Tropea, Collision Phenomena in Liquids and Solids (Cambridge University Press, Cambridge, 2017).

[17] F. R. Smith, N. C. Buntsma, and D. Brutin, Roughness influence on human blood drop spreading and splashing, Langmuir (2017).

[18] F. Ramsthaler, J. Schlote, C. Wagner, J. Fiscina, and M. Kettner, The ring phenomenon of diluted blood droplets, Int. J. Legal Med. 130, 731 (2016).

[19] R. D. Deegan, O. Bakajin, T. F. Dupont, G. Huber, S. R. Nagel, and T. A. Witten, Capillary flow as the cause of ring stains from dried liquid drops, Nature (London) 389, 827 (1997).

[20] J. B. Lee, D. Derome, R. Guyer, and J. Carmeliet, Modeling the maximum spreading of liquid droplets impacting wetting and nonwetting surfaces, Langmuir 32, 1299 (2016).

[21] N. Kabaliuk, M. Jermy, K. Morison, T. Stotesbury, M. Taylor, and E. Williams, Blood drop size in passive dripping from weapons, Forensic Sci. Int. 228, 75 (2013).

[22] R. Sahu, S. Sett, A. Yarin, and B. Pourdeyhimi, Impact of aqueous suspension drops onto non-wettable porous membranes: Hydrodynamic focusing and penetration of nanoparticles, Colloids Surf. A 467, 31 (2015).

[23] L. Xu, W. W. Zhang, and S. R. Nagel, Drop Splashing on a Dry Smooth Surface, Phys. Rev. Lett. 94, 184505 (2005).

[24] A. K. Martusevich, Y. Zimin, and A. Bochkareva, Morphology of dried blood serum specimens of viral hepatitis, Hepat. Mon. 7, 207 (2007). 